\title{
Nanostructural Features of Silver Nanoparticles Powder Synthesized through Concurrent Formation of the Nanosized Particles of Both Starch and Silver
}

\author{
A. Hebeish, M. H. El-Rafie, M. A. El-Sheikh, and Mehrez E. El-Naggar \\ Textile Research Division, National Research Centre, Dokki, P.O. Box 12622, Giza 12522, Egypt
}

Correspondence should be addressed to Mehrez E. El-Naggar; mehrez_chem@yahoo.com

Received 25 June 2013; Revised 31 July 2013; Accepted 2 August 2013

Academic Editor: Xiao Wei Sun

Copyright (C) 2013 A. Hebeish et al. This is an open access article distributed under the Creative Commons Attribution License, which permits unrestricted use, distribution, and reproduction in any medium, provided the original work is properly cited.

\begin{abstract}
Green innovative strategy was developed to accomplish silver nanoparticles formation of starch-silver nanoparticles (St-AgNPs) in the powder form. Thus, St-AgNPs were synthesized through concurrent formation of the nanosized particles of both starch and silver. The alkali dissolved starch acts as reducing agent for silver ions and as stabilizing agent for the formed AgNPs. The chemical reduction process occurred in water bath under high-speed homogenizer. After completion of the reaction, the colloidal solution of AgNPs coated with alkali dissolved starch was cooled and precipitated using ethanol. The powder precipitate was collected by centrifugation, then washed, and dried; St-AgNPs powder was characterized using state-of-the-art facilities including UV-vis spectroscopy, Transmission Electron Microscopy (TEM), particle size analyzer (PS), Polydispersity index (PdI), Zeta potential (ZP), XRD, FT-IR, EDX, and TGA. TEM and XRD indicate that the average size of pure AgNPs does not exceed $20 \mathrm{~nm}$ with spherical shape and high concentration of AgNPs (30000 ppm). The results obtained from TGA indicates that the higher thermal stability of starch coated AgNPS than that of starch nanoparticles alone. In addition to the data obtained from EDX which reveals the presence of AgNPs and the data obtained from particle size analyzer and zeta potential determination indicate that the good uniformity and the highly stability of St-AgNPs).
\end{abstract}

\section{Introduction}

A nanoscale metal is defined as a metal that has a structure in the nanometer size range, usually ranging from 1 to $100 \mathrm{~nm}$. Of these metal nanoparticles, silver nanoparticles (AgNPs) have been by far the most important both from scientific and practical points of view by virtue of their potential use in industrial and other applications [1].

Intensive research has been focused on AgNPs due to their unique optical electronic, $[2,3]$ catalytic, [4-6], and antimicrobial [7-9] properties which are greatly different from bulk substances. Such properties strongly depend on size and shape of AgNPs as well as on their interactions with the stabilizing agent and the surrounding media in addition to the manner of their preparation. Thus, the controllable synthesis of the nanocrystals is a key challenge to achieve their better applied characteristics [10].
AgNPs have been synthesized using various methods. The physicochemical methods (e.g., ultrasound, templates, and milling process [11-13]), as well as "green" synthesis using microorganisms, enzymes, plants, or plant extracts [14-16] are equally effective, but sometimes they require complex apparatus [17]. Synthesis of AgNPs as per the chemical reduction in aqueous and nonaqueous solutions is the most common owing to its convenience, facility, being not timeconsuming, and producing a high yield of the nanoparticles. Furthermore, the nanoparticles obtained by chemical route can be stored for a long time without losing their stability.

Chemical reduction process for a typical synthesis of AgNPs process consists of three main stages. The first stage is the reduction of a silver salt by a reducing agent. $\mathrm{AgNO}_{3}$ is the most frequently employed as a source of silver ions. In the second stage, neutral silver atoms are formed and collide with each other, forming stable nuclei, and a growth 
of the nanoparticle occurs until all metal ions are consumed. The third stage entails prevention of nanoparticles agglomeration by an addition of capping agent which stabilizes the formed AgNPs and prevents these nanoparticles from agglomeration via their interactions with small particles [18].

In some methods used for the preparation of AgNPs, some toxic chemicals were used as a reducing agent such as sodium borohydride, [19] citrate, or [20] N, N dimethyl formamide [21], and hydrazine hydrate [22]. Such reducing agents may be associated with environmental toxicity or biological hazards, a problem which pursues the development of a green synthesis for AgNPs. Nontoxic chemicals and renewable materials are preferably used because of their advantage in reducing the environmental risk.

Raveendran et al. [23] were the first to develop the concept of green synthesis of nanoparticles. They used $\beta$ D-glucose as the reducing agent and starch as a capping agent to prepare silver nanoparticles. Natural polysaccharides such as chitosan [24] and cellulose [25] were also employed for preparation of AgNPs. Previous reports disclosed also the well-stabilized, highly distributed and size-controlled of AgNPs solution with diameters ranging from 10 to $15 \mathrm{~nm}$, produced using hydroxypropyl starch (HPS) as reducing agent for silver nitrate as well as stabilizing agent for the formed AgNPs [26].

The present work is targeted to accomplish AgNPs through green innovative strategy. The innovation is based on synthesis of nanoparticles of both starch and silver concomitantly in one single step. Once this is the case, starch nanoparticles will perform dual role: as a reducing agent for silver ions and a capping agent for the silver nanoparticles formed thereof with production of starch-silver nanoparticles (St-AgNPs). Nanoprecipitation method issued and the resultant St-AgNPs. St-AgNPs are anticipated to be achieved in the powder form. Another target of current work is to obtain St-AgNPs with AgNPs contents along with controllable size and size distriburion that advocate them for industrial and other applications. Highly sophisticated tools for analyzing, characterization, and aessessment of the nanostructural features of St-AgNPs.

\section{Materials and Methods}

2.1. Materials. All chemicals used in this investigation were reagent-grade materials and they were used without purification.

Native maize starch (NS) was purchased from the Egyptian Company for Starch and Glucose Manufacture, Cairo, Egypt. Silver nitrate $\left(\mathrm{AgNO}_{3} ; 99 \%\right)$, sodium hydroxide, and absolute ethyl alcohol were of analytical grade (99.5\%). All aqueous solutions were made using distilled water.

\subsection{Methods}

2.2.1. Preparation of Powdered St-AgNPs. In the process of StAgNPs preparation by the chemical reduction method, alkali dissolved starch was used as reducing agent for silver ions and stabilizing agent for the formed AgNPs. Preparation of
St-AgNPs was carried out in thermostatic water bath and high-speed homogenizer. A solution of alkali dissolved starch was prepared by placing $5 \mathrm{~g}$ of native maize starch in $80 \mathrm{~mL}$ of distilled water containing $1.5 \mathrm{~g}$ sodium hydroxide using high-speed homogenizer until complete dissolution. After complete dissolution, the temperature raised to $70^{\circ} \mathrm{C}$. Six different amounts of $\mathrm{AgNO}_{3}(0.175,0.785,1.57,3.14,4.72$, and $6.28 \mathrm{~g}$ ) dissolved in $20 \mathrm{~mL}$ distilled water were added dropwise to the aqueous solution of alkali dissolved starch at $\mathrm{pH}$ 12. The reaction mixture was held under continuous stirring for $60 \mathrm{~min}$. Short time after the addition of $\mathrm{AgNO}_{3}$ solution, the reaction medium acquired a clear yellow colour then changed to brown and finally to dark brown colour showing the formation of St-AgNPs. After complete reaction, the colloidal solution was allowed to cool down slowly to $25^{\circ} \mathrm{C}$ within $30 \mathrm{~min}$. Then the St-AgNPs was precipitated using absolute ethyl alcohol under high-speed homogenizer. The powder precipitate was collected by centrifugation at $4,500 \mathrm{rpm}$ for $15 \mathrm{~min}$, washed twice with 80/20 ethanol/water to remove the unreacted materials and impurities, and then finally washed with absolute ethanol. The collected powder was dried and identified as St-AgNPs using state-of-the-art facilities.

It is expected theoretically that when the as prepared powder redispersed in $100 \mathrm{~mL} \mathrm{H}_{2} \mathrm{O}$ will retain colloidal solution containing St-AgNPs having concentrations of 1000, 5000, 10000, 20000, 30000 and 40000 ppm.

\subsubsection{Characterization}

Ultraviolet Visible (UV-Vis) Spectroscopy. The formation of reduced AgNPs in colloidal solution was monitored using UV-vis spectral analysis. Color changes in the supernatant were monitored both by visual inspection and absorbance measurements using T80 spectrophotometer. The spectra of the surface plasmon resonances of AgNPs were recorded using a UV-Vis spectrophotometer at wavelengths between 250 and $600 \mathrm{~nm}$. All samples solutions were diluted $1: 10$.

Transmission Electron Microscopy (TEM). The morphological examination of the nanoparticles was performed by transmission electron microscopy (TEM) on a JEOL (JEM-1010) instrument with an acceleration voltage of $80 \mathrm{kV}$ after drying of a drop of aqueous AgNPs on a carbon-coated copper TEM grid.

The particle size distribution of the AgNPs obtained from TEM images was evaluated using Image J 1.45s software.

Hydrodynamic Size and Zeta Potential Measurement of AgNPs. The average particle size along with its polydispersity index (PdI) and the zeta potential (ZP) of the nanoparticles was analysed by photon correlation spectroscopy and laser Doppler anemometry, respectively, using a Zetasizer Nano ZS (Malvern Instruments, UK). Prepared nanoparticles were separated and subjected to measurement following dilution with $0.45 \mu \mathrm{m}$ filtered distilled water. Particle size and PDI measurements were performed at a scattering angle of $90^{\circ}$ and at a temperature of $25^{\circ} \mathrm{C}$. The hydrodynamic diameter was calculated from the autocorrelation function of the intensity 
of light scattered from particles with the assumption that the particles had a spherical form. The samples for ZP were placed in a disposable zeta cell at a temperature of $25^{\circ} \mathrm{C}$ and were measured using PALS technology. The measurement was repeated three times for each sample.

Fourier Transform Infrared (FT-IR) Spectroscopy. FTIR spectra of the Starch containing AgNPs compared with those of native maize starch were obtained using Nicolet 6700, Thermo Scientific, MA, USA. A $1 \%$ of each dried samples was mixed with $\mathrm{KBr}$ and compressed to form tablets. The IR spectra were scanned over the wave number range of 4000 $400 \mathrm{~cm}^{-1}$.

$X$-Ray Diffractometry (XRD). X-ray diffraction (XRD) patterns of finely powdered samples were recorded on a Philips PW3040 X-Ray diffractometer system by monitoring the diffraction angle from $5^{\circ}$ to $80^{\circ}(2 \theta)$ at $40 \mathrm{keV}$. The average crystallite size AgNPs, $D$, can be calculated using from the line broadening using the Scherrer's relation:

$$
D_{\mathrm{hkl}}=\frac{(K \times \lambda)}{\left(\beta_{\mathrm{hkl}} \times \cos \theta_{\mathrm{hkl}}\right)},
$$

where $D_{\mathrm{hkl}}$ is the particle size perpendicular to the normal line of (hkl) plane, $k$ is a constant $(0.9), \beta_{\mathrm{hkl}}$ is the full width at half maximum of the (hkl) diffraction peak, $\theta_{\mathrm{hkl}}$ is the Bragg angle of (hkl) peak, and $\lambda$ is the wavelength of $\mathrm{X}$-ray.

Energy Dispersive X-Ray Analysis (EDX). The elemental analysis was performed using EDX, which is an attachment to the scanning electron microscopy. The sample powder of AgNPs was compressed to form tablets before analysis with EDX spectrum.

Atomic Absorption Spectroscopy (AAS). The concentration of silver was determined by the AAS method with an Analyst 300 Perkin-Elmer spectrometer. The as synthesized and concentrated solution of silver was used as a reference sample. The AAS was performed at wavelength $328.1 \mathrm{~nm}$.

\section{Results and Discussion}

As already stated, the present work introduces an innovation which is based on concurrent synthesis of nanoparticles of both starch and silver in a single-stage process. The alkali dissolved starch act as reductant for silver ions and stabilizer for the AgNPs formed thereof. The function of sodium hydroxide is twofold: first, to dissolve the native starch and second, to adjust medium $\mathrm{pH}$ up to 12 which leads to acceleration in the formation of AgNPs. The end reducing group at C6 of the starch molecules is looked upon as the reducing agent for silver ions. Ag ions reduce the influence of alkali dissolved starch. It is logical, however, that alkali dissolved starch can coordinate $\mathrm{Ag}$ ions before reduction and, in so doing, form a polymer-metal ion complex. Such a complex undergoes reduction under mild conditions, bringing about a smaller size and a narrow size distribution of the nanoparticles. After reduction, the stabilizing effect of these macromolecules is a manifestation of either that particles are attached to much larger protecting polymer or the protecting polymer covers that or encapsulates AgNPs [23].

It is as well to emphasize that we here used the precipitation reduction method due to its advantages, namely, the process is simple, the resulting system is stable, and the concentration of silver in the resulting system can be comparatively higher than that in silver colloids prepared by other methods. It is, therefore, envisaged that the method in question may provide more opportunities for feasible mass production of AgNPs powder.

The addition of small amount of diluted solution of $\mathrm{NaCl}$ or $0.01 \mathrm{~N} \mathrm{HCl}$ to the remained supernatant after centrifugation confirmed the full reduction for Ag ions to AgNPs (no white precipitate formed).

Obviously, then, the ultimate product of concurrent synthesis of St-NPs and AgNPs is St-AgNPs. Detailed characteristics of the nanostructural features of St-AgNPs are given in the next paragraph.

3.1. UV-Visible and TEM Analysis of AgNPs. During the synthesis of St-AgNPs, yellow, brown, and light brown colours have appeared in solution along with mirror-like illumination on the walls of the Erlenmeyer flask. This clearly indicates the formation of AgNPs in the reaction mixture. The colour of the solution was due to the excitation of surface plasmon vibrations in the AgNPs.

The AgNPs were characterised by UV-vis spectroscopy which seems to be very useful technique in the analysis of nanoparticles. Figure 1 shows the UV-Vis spectroscopy of alkali dissolved starch and alkali dissolved starch coated AgNPs. It is worthy to observe that the alkali dissolved starch has no characteristic peak. Upon the addition of $\mathrm{AgNO}_{3}$ $(0.175 \mathrm{~g})$, a small peak is observed at $402 \mathrm{~nm}$ indicating the formation of AgNPs. Prolonging the concentration of $\mathrm{AgNO}_{3}$ from $0.175 \mathrm{~g}$ to 0.785 leads to marginal increase of the peak intensity with marginal shift indicating the formation of AgNPs with higher concentration and still in the smallest diameter. On further addition of different concentrations of $\mathrm{AgNO}_{3}(1.75,3.1$, or $4.72 \mathrm{~g})$, the peaks intensity increases with small shift and reaches $417 \mathrm{~nm}$ when the concentration of $\mathrm{AgNO}_{3}$ reaches the highest concentration of $\mathrm{AgNO}_{3}(4.72 \mathrm{~g})$. This means that the Ag ions are converted to AgNPs and the peak heights are then directly related to the number of AgNPs present in the solution. Further increase in the concentration of $\mathrm{AgNO}_{3}$ up to $6.28 \mathrm{~g}$ is accompanied by unexpected enhancement in the absorption intensity and shifts the band towards longer wavelength $(452 \mathrm{~nm})$ which could be attributed to enlargement in the AgNPs size. In addition to the appearance of clearly peak at $350 \mathrm{~nm}$ when the concentration of $\mathrm{AgNO}_{3}$ is $6.28 \mathrm{~g}$, indicating the presence of $\mathrm{Ag}$ ions (not reduced) due to the concentration of alkalidissolved starch is not more enough to reduce all the highest concentrations of $\mathrm{Ag}$ ions [26].

We can see from Figure 1 that the UV spectra of AgNPs solutions of different concentrations (1000, 5000, 10000, 20000,30000 and $40000 \mathrm{ppm}$ ) have only an absorption peak ranging from 402 to $417 \mathrm{~nm}$ for AgNPs at concentrations ranging from 1000 to $30000 \mathrm{ppm}$. While in the case of AgNPs 


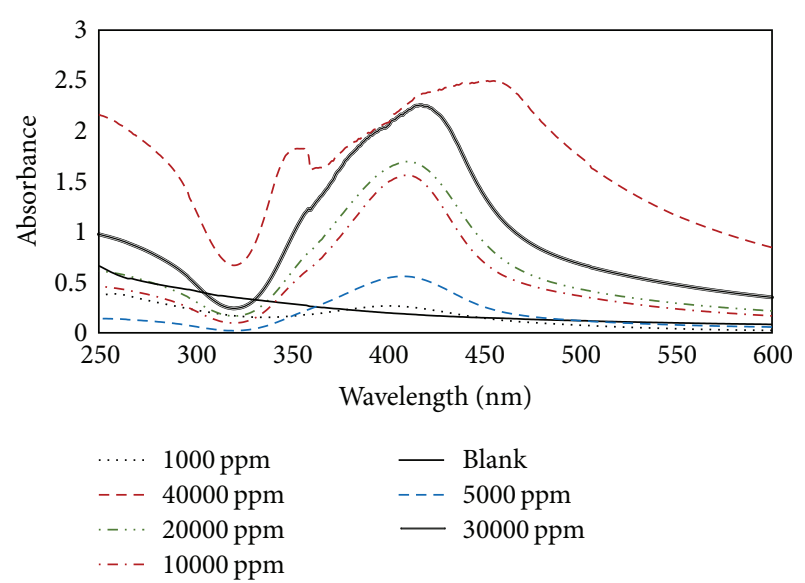

FIgURE 1: UV-visible spectra of St-NPs and AgNPs at different concentrations (1000, 5000, 10000, 20000, 30000, and $40000 \mathrm{ppm})$.

with $40000 \mathrm{ppm}$, new absorption peak occurs for silver ions at wavelength $356 \mathrm{~nm}$.

The primary study shows that the absorption intensity increases as the concentration of $\mathrm{AgNO}_{3}$ increases, which reflects in the formation of more Ag nanoparticles. At a low concentration of $\mathrm{AgNO}_{3}$, the maximum absorption wavelength gives rise to a blue shift, meaning a decrease in the particles size.

Table 1 depicts the actual concentration of AgNPs obtained with different concentrations of $\mathrm{AgNO}_{3}$ reduced by alkali dissolved starch which was evaluated by AAS and the maximum wavelength monitored from UV-visible spectroscopy.

It was found that the concentration of AgNPs in all samples was lower than the theoretical (calculated) value.

It is further noted that increasing the concentration of silver nitrate gradually during the preparation of powdered AgNPs led to red shift of UV spectra indicating that the particles' size increased and that the large silver aggregates were formed especially with the huge increase in the case of $40000 \mathrm{ppm}$ which attained the maximum absorbance at $452 \mathrm{~nm}$.

The silver nanoparticles were further investigated by TEM and their size distributions were measured image J 1.45 s software.

Figures 2 and 3 contain a typical TEM micrograph and the particle size distribution of St-AgNPs being calculated from AgNPs component concentrations (1000, 5000, 10000, 20000, 30000, and $40000 \mathrm{ppm}$ ) where the resultant powder obtained is dissolved in $100 \mathrm{~mL} \mathrm{H}_{2} \mathrm{O}$. The TEM figures out the spherical morphology of the formed St-AgNPs as displayed in Figures 2(a), 2(c), and 2(e) and Figures 3(a) and 3(c). It was observed that the size of the colloidal solution of AgNPs coated with alkali dissolved starch have small size with marginal increase by increasing the concentration of $\mathrm{Ag}$ ions in the powder up to $30000 \mathrm{ppm}$. Further increase in the $\mathrm{Ag}$ ions up to $40000 \mathrm{ppm}$ led to highly aggregated products without complete reduction of $\mathrm{Ag}$ ions.
A close examination of Figures 2(b), 2(d), and 2(f) and Figures 3(b) and 3(d) would reveal that the predominant AgNPs size lies between 7.53 and $21.060 \mathrm{~nm}$ and there is marginal ascending in the mean particles size which is nearly undetectable; this phenomenon correlates with the increase in the amount of primary $\mathrm{AgNO}_{3}$. However, this interpretation is signed to not affect on the features of the StAgNPs.

3.2. Electron Diffraction Pattern of Silver Nanoparticles. The crystallinity of the AgNPs was detected by selected area diffraction (SAD) experiments, and a typical SAD pattern is shown in Figure 4(a). When the electron diffraction is carried out on a limited number of crystals, the appearance of discrete spots in the ring pattern proved that the majority of the particles are single crystalline materials and they are predominately oriented along their Ag direction, as commonly found for the fcc silver crystal lattice [27]. The interplanar spacing determined from the rings of the diffraction patterns showing the plane distances $2.3 \AA, 2.04 \AA, 1.39 \AA$, and $1.27 \AA$ are consistent with the plane families of pure face-centred cubic (fcc) silver structure (Figure 4(b)).

3.3. Particle Size (PS) Analyser, Polydispersity Index (PdI), and Zeta Potential (ZP) of AgNPs. Mean PS diameter and PdI were all measured in solutions using dynamic light scattering (DLS). The size of the colloidal AgNPs and their granulometric are disclosed (Figure 5(a)). As is evident, the size of the particles is between 5 and $50 \mathrm{~nm}$. PS analyzer displays the presence of AgNPs with low PdI (0.163).

Figure 5(b) shows the ZP measurement of AgNPs at concentration equal to $30000 \mathrm{ppm}$ in a solution form. For the obtained AgNPs, ZP value was measured and found to be $-28 \mathrm{mV}$. The negative $\mathrm{ZP}$ of the synthesized AgNPs was due to the capping of the particles by the hydroxyl groups of starch molecules. Current data are in conformation with the literature; solutions with $\mathrm{ZP}$ above $+20 \mathrm{mV}$ or below $-20 \mathrm{mV}$ are considered stable [28]. The average size and PdI determined by DLS are in concurrence with the the results of TEM image and the particle size distribution evaluated using Image J $1.45 \mathrm{~s}$ software. By studying the dynamic light scattering, it is clear that the AgNPs exhibit a narrow size distribution for the nanoparticles.

The said value of $\mathrm{ZP}$ provides full stabilization of the AgNPs, which may be the main reason in producing particle sizes with a narrow size distribution index. The value of the ZP of the St-AgNPs in addition to their narrow size distributions provides satisfactory evidence about their little tendency towards aggregation.

Such behaviour suggests unambiguously the presence of strong electric charges on the particles' surfaces to hinder agglomeration. The electric changes were found to fall in the negative side, which reflect the efficiency of the capping materials in stabilizing the nanoparticles by providing intensive negative charges that keep all the particles away from aggregation. The electric charges were strong enough to hinder agglomeration and to provide stabilization of the nanoparticles. It follows from this that the AgNPs particles 
TABle 1: Actual concentration (AAS), $\lambda_{\max }$ of AgNPs.

\begin{tabular}{lcccccc}
\hline $\begin{array}{l}\text { AgNPs theoretical concentration, } \\
\text { ppm }\end{array}$ & 1000 & 5000 & 10000 & 20000 & 30000 & 40000 \\
$\begin{array}{l}\text { AgNPs concentration (AAS } \\
\text { method) }\end{array}$ & 989.31 & 4902 & 9693.79 & 18138.034 & 26322.53 & - \\
AgNPs conc. \% actual, & 98.931 & 98.04 & 96.937 & 90.69 & 87.74 & - \\
$\lambda_{\max }$ & $402 \mathrm{~nm}$ & $408 \mathrm{~nm}$ & $411 \mathrm{~nm}$ & $413 \mathrm{~nm}$ & $417 \mathrm{~nm}$ & $452 \mathrm{~nm}$ \\
\hline
\end{tabular}

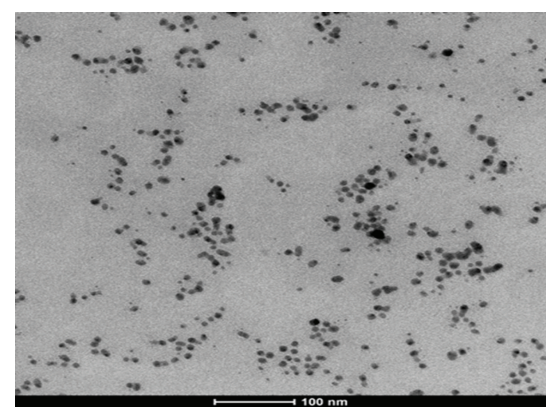

(a)

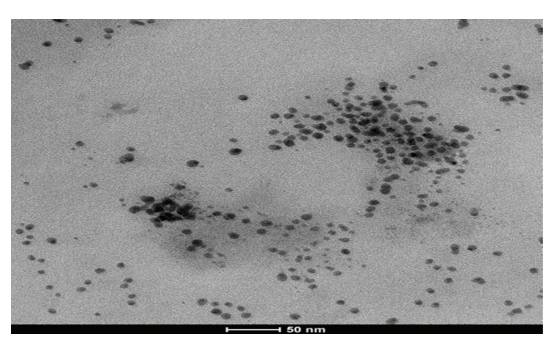

(c)

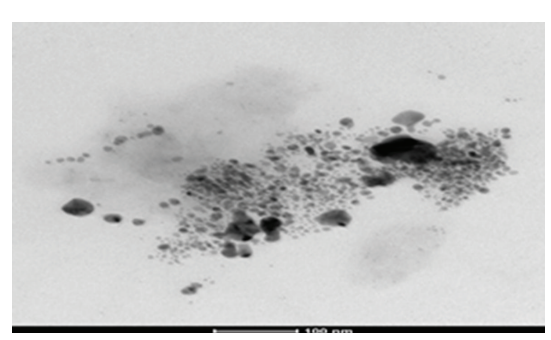

(e)

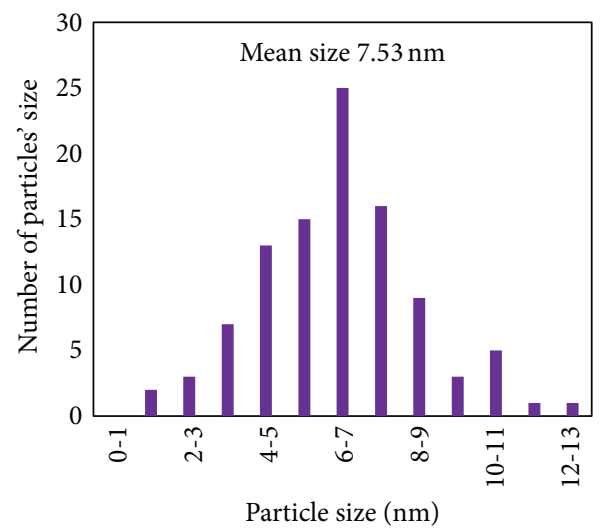

(b)

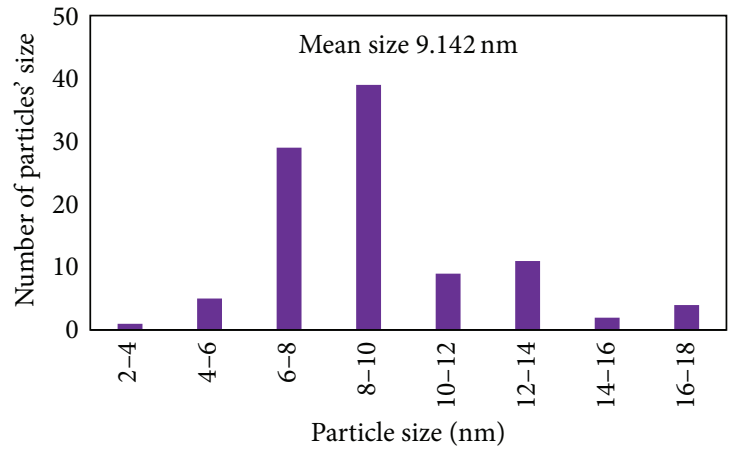

(d)

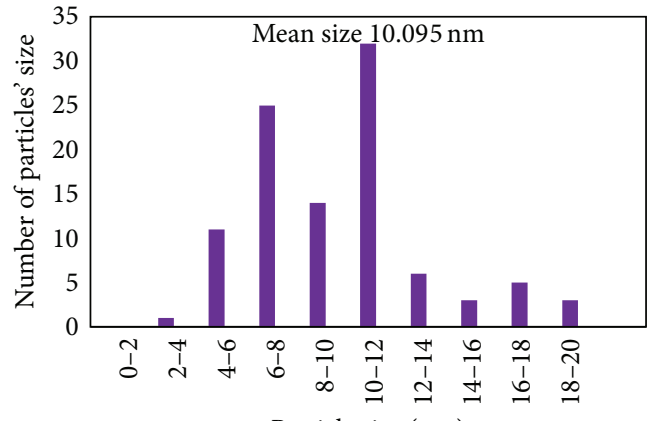

Particle size (nm)

(f)

Figure 2: TEM micrographs and histogram of silver colloids with different concentrations of AgNPs (ppm) prepared at different concentrations of silver nitrate. (a), (b) $1000 \mathrm{ppm}$. (c), (d) $5000 \mathrm{ppm}$; (e), (f) $10000 \mathrm{ppm}$; Applied condition: $5 \mathrm{gm} \mathrm{NS}$; pH 12; time 60 min; temp. $70^{\circ} \mathrm{C}$; different amounts of $\mathrm{AgNO}_{3}(0.175,0.785$, and $1.57 \mathrm{~g})$; total volume, $100 \mathrm{~mL} \mathrm{H}_{2} \mathrm{O}$. 


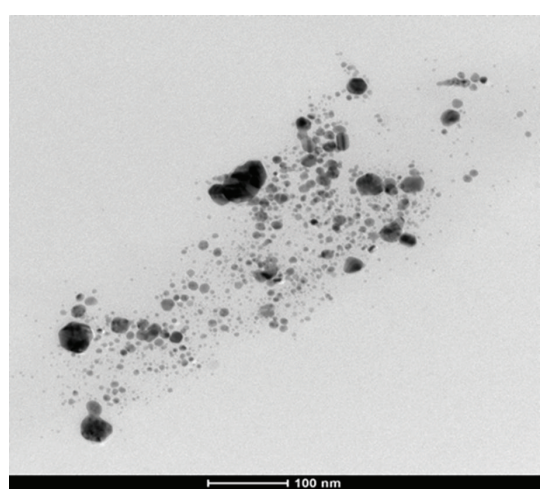

(a)

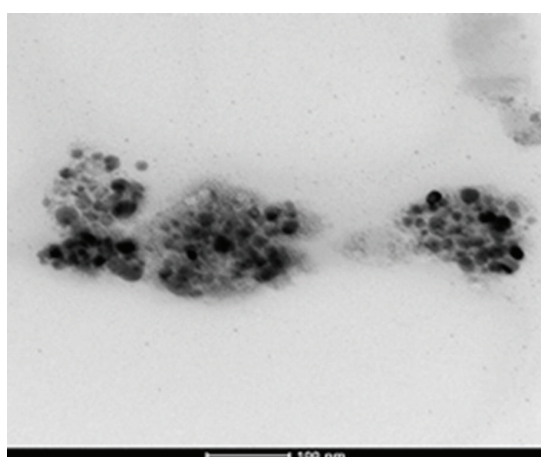

(c)

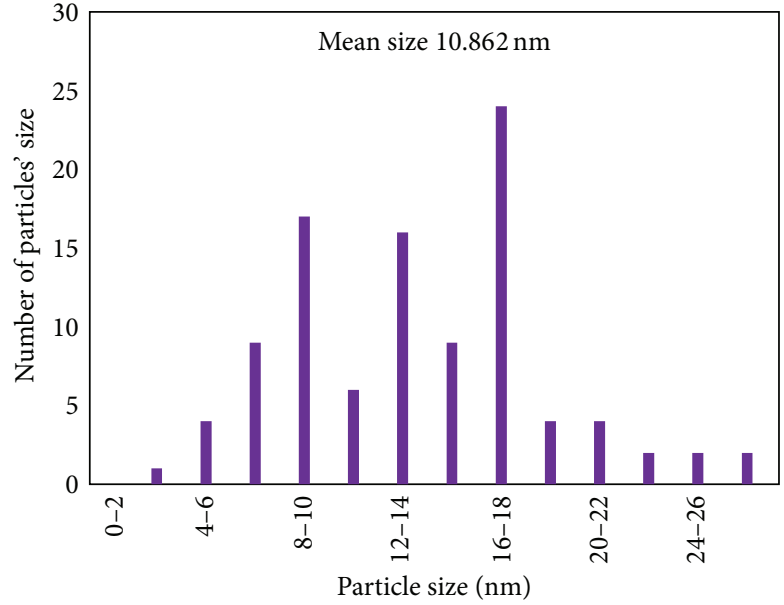

(b)

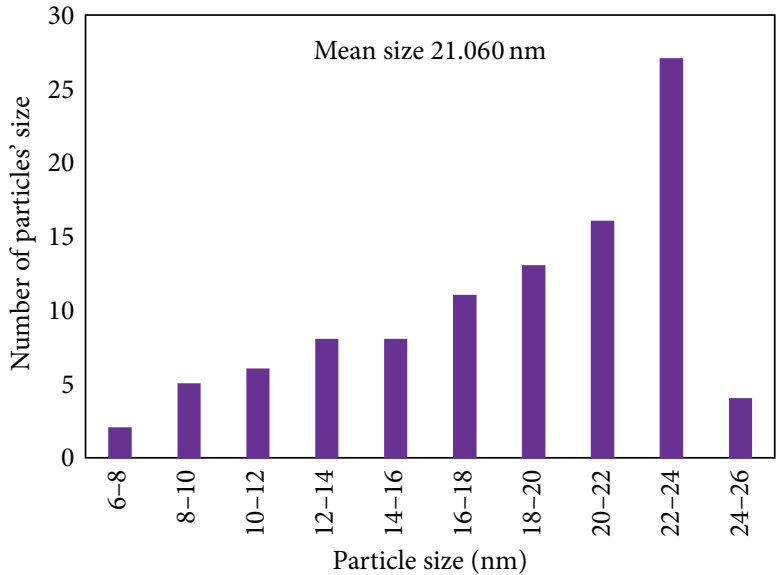

(d)

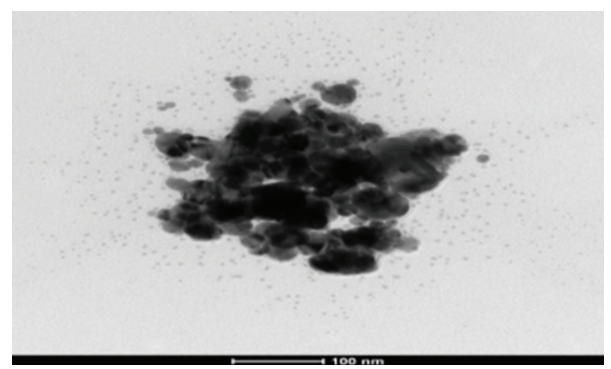

(e)

FIGURE 3: TEM and histogram of silver colloids having different concentrations of AgNPs (ppm) prepared using different concentrations of silver nitrate. (a), (b); 20000 ppm; (c), (d); 30000 ppm; (e) 40000 ppm. Applied condition: 5 gm NS; pH 12; time $60 \mathrm{~min}$; temp. $70^{\circ} \mathrm{C}$; different amounts of $\mathrm{AgNO}_{3}$ (3.14, 4.72, and $6.28 \mathrm{~g}$ ); total volume, $100 \mathrm{~mL} \mathrm{H} \mathrm{H}_{2} \mathrm{O}$.

and thus their solution are stable, which is also in accordance with the result previously reported before for colloidal nanoparticles stability [29].

3.4. FT-IR Studies of St-NPs and St-AgNPs. Evidence for the interaction of hydroxyl groups of starch with Ag metal in the stabilization of the silver clusters is obtained from the Fourier transform infrared (FT-IR) spectra of the alkali dissolved starch-containing Ag clusters (Figure 6). The FTIR spectra of St-NPs alone exhibited characteristics bands at 3435.7 and $2926,43 \mathrm{~cm}^{-1}$ because of the $\mathrm{O}-\mathrm{H}$ stretching vibrations of the starch associated with $\mathrm{C}-\mathrm{H}$ stretching vibrations. Additional characteristics absorption bands appeared at $1026 \mathrm{~cm}^{-1}$ because of $\mathrm{O}-\mathrm{H}$ bends vibrations, respectively. The band at $3435.7 \mathrm{~cm}^{-1}$ and 1026 shifted to $3426 \mathrm{~cm}^{-1}$ and $1004.23 \mathrm{~cm}^{-1}$, respectively, in the presence of AgNPs; also, 


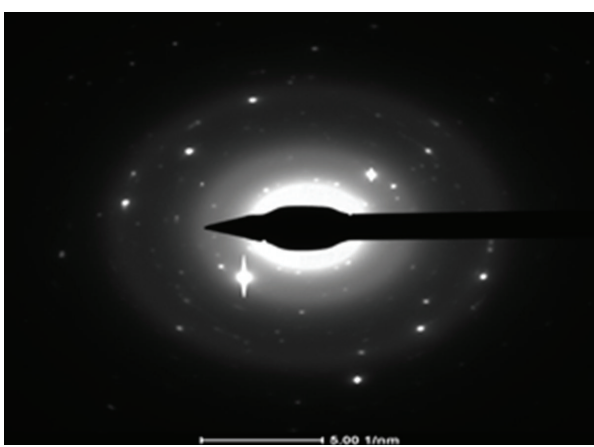

(a)

\begin{tabular}{ccc}
\hline Number & Experimental $d(\AA)$ & Cubic Ag \\
\hline 1 & 111 & 2.30 \\
\hline 2 & 200 & 2.04 \\
\hline 3 & 220 & 1.39 \\
\hline 4 & 311 & 1.27 \\
\hline
\end{tabular}

(b)

FIGURE 4: (a) Electron diffraction pattern of silver nanoparticles (b) interplanar spacing determined from the rings of the diffraction patterns.

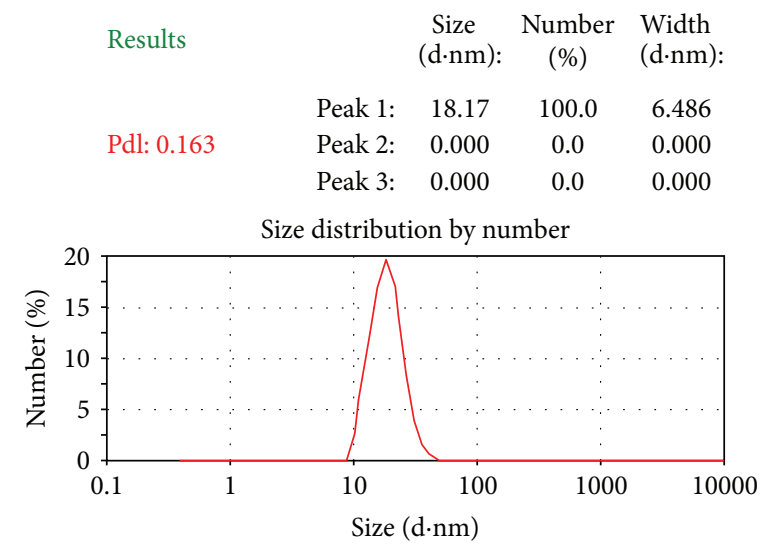

(a)
Results

$\zeta$ deviation $(\mathrm{mV}): 4.16$

Conductivity (mS/c): 0.0152
Mean Area Width

$(\mathrm{mV}) \quad(\%) \quad(\mathrm{mV})$

Peak 1: $\quad-28.4 \quad 100.0 \quad 4.22$

$\begin{array}{llll}\text { Peak 2: } & 0.00 & 0.0 & 0.00\end{array}$

$\begin{array}{llll}\text { Peak 3: } \quad 0.00 & 0.0 & 0.00\end{array}$

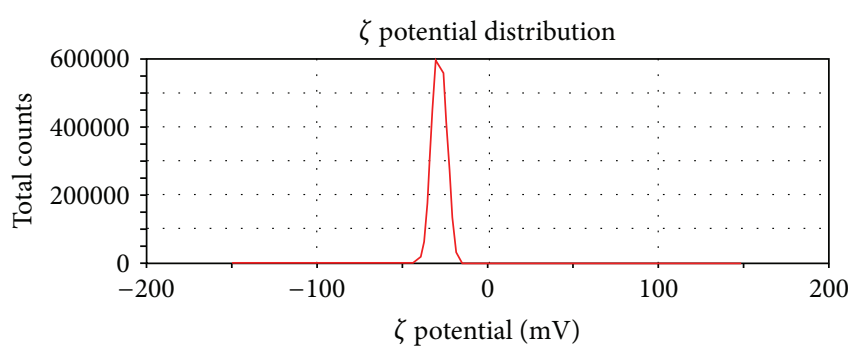

(b)

FIgURE 5: (a) The average size of the AgNPs with the polydispersity index (PdI); (b) the average ZP of AgNPs.

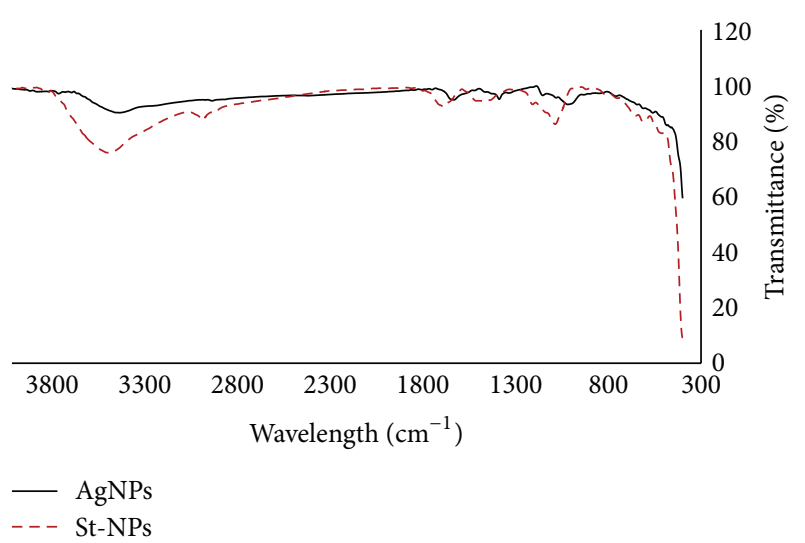

FIGURE 6: FT-IR of St-NPs and St-AgNPs.

the band was broader in the precipitated St-NPs containing AgNPs compared to St-NPs.

These observations clearly show the interaction of $\mathrm{Ag}$ with the $\mathrm{OH}$ group of St-NPs. The polar groups O-H of StNPs have the good ability of coordination reaction with metal ions (e.g., with $\mathrm{Ag}$ ions). When $\mathrm{O}-\mathrm{H}$ groups and $\mathrm{Ag}$ ions form coordination bonds, the interactions among the resultant Ag

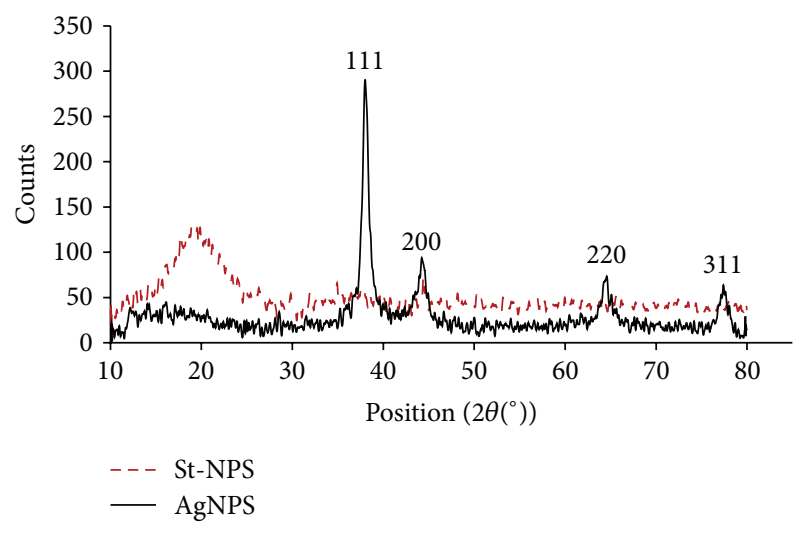

FIGURE 7: XRD of St-NPs and nano-precipitated starch coated AgNPs.

particles and oxygen atoms of $\mathrm{O}-\mathrm{H}$ groups become stronger with increasing amount of Ag. This can lead to corresponding changes both in the positions and in the strengths of FT-IR spectra of St-NPs. Thus, the stretching peaks of both $\mathrm{O}-\mathrm{H}$ groups become wider gradually [30]. 


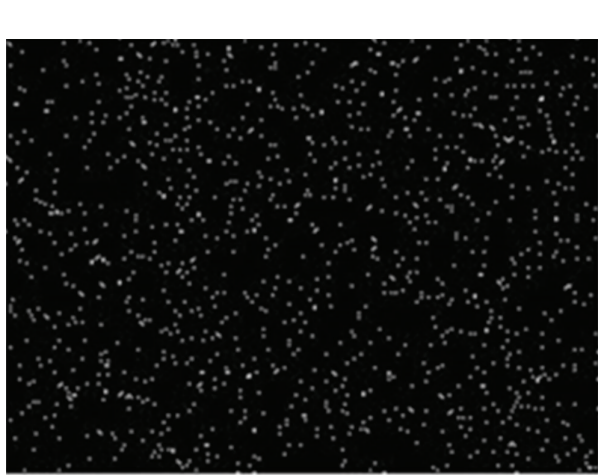

Ag Lal

\begin{tabular}{|l|l|l|}
\hline Element & Weight & Atomic (\%) \\
\hline C K & 30.47 & 48.02 \\
\hline O K & 38.24 & 45.24 \\
\hline Na K & 1.92 & 1.58 \\
\hline Ag L & 29.38 & 5.16 \\
\hline Totals & 100.00 & \\
\hline
\end{tabular}

(a)

(b)

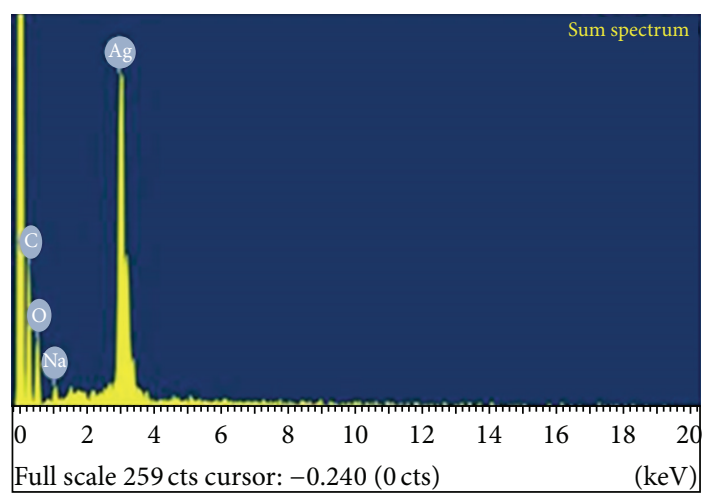

(c)

FIgure 8: (a) EDX mapping of AgNPs powder, (b) EDX quantitative analysis, and (c) EDX spectra of AgNPs.

All the other peaks of St-NPs almost maintained their position in the corresponding St-AgNPs sample, but with a change in their peak intensity which indicated that StNPs adsorbed on the silver particles through static electricity gravitation.

3.5. XRD Studies of AgNPs. X-ray diffraction (XRD) was performed to ascertain the identity of the final products. The XRD results of St-NPs and AgNPs precipitated with St-NPs are shown in Figure 7. As is evident, the St-NPs have one strong characteristic peak at $20.054^{\circ}$ while in the case of AgNPs coated with St-NPs, the four diffraction peaks obtained at angles $2 \theta$ of $38.1952^{\circ}, 44.2987^{\circ}, 64.4087^{\circ}$, and $77.6079^{\circ}$, respectively, are attributed solely to the face-centred cubic (fcc) crystalline silver content of the sample. These peaks correspond to the four d-spacings (111), (200), (220), and (311), respectively. The diffracted peaks of the face centered cubic crystalline were matched with the Joint Committee on Powder Diffraction Standards (JCPDS) File no. 03-0921 (JCPDS, no. 4-0783). Furthermore, no additional peaks in the $\mathrm{XRD}$ were observed for $\mathrm{Ag}_{2} \mathrm{O}$ revealing the high purity of the as synthesized silver crystal.

It is as well to report that, the average particle size of AgNPs at the optimum conditions for preparation according to Scherrers equation calculated using the width of the (111) peak is estimated to be $20.3 \mathrm{~nm}$ which was nearly in consonance with the particle size obtained from TEM image and particle size analyzer AgNPs.

3.6. Energy Dispersive X-Ray Spectra (EDX) of AgNPs. Chemical analysis of the produced AgNPs was accomplished by means of EDX, which confirmed both the existence of Ag and the Alkali dissolved starch that covers the AgNPs; the latter is implied by the presence of the $\mathrm{C}, \mathrm{O}$, and $\mathrm{Na}$ peaks in the EDX spectra (Figure 8(c)). Metallic silver nanocrystals generally show typical optical observation peak approximately at $3 \mathrm{keV}$ due to surface plasmon resonance. The EDX spectra also proved that the Ag nanoparticles are in metallic form, with no formation of $\mathrm{Ag}_{2} \mathrm{O}$ in them and free from any other impurities.

Figure 8(a) shows the elemental map of AgNPs. The results indicate that AgNPs are homogeneous and stabilized by alkali dissolved starch. The table shown in Figure 8(b) confirmed the presence of powdered AgNPs at high concentration.

3.7. TGA of St-NPs and St-AgNPs. Figure 9 shows the thermograms of St-NPs and St-AgNPs. TGA spectra have been recorded in temperature range from $25^{\circ} \mathrm{C}$ to $500^{\circ} \mathrm{C}$ using simultaneous thermal system (Shimadzu, DTG-60). A ceramic $\left(\mathrm{Al}_{2} \mathrm{O}_{3}\right)$ crucible was used for heating and measurements were carried out in nitrogen atmosphere at the heating 


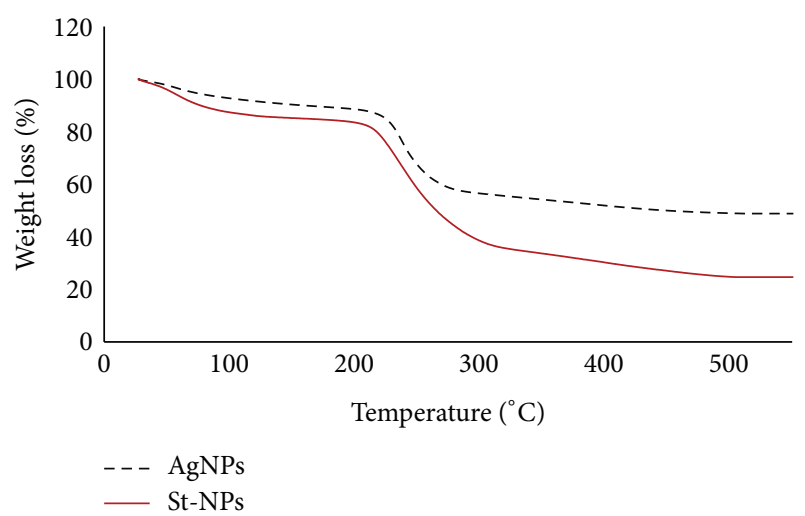

Figure 9: TGA of St-NPs and AgNPs.

rate of $10^{\circ} \mathrm{C} / \mathrm{min}$. The thermal stability of AgNPs was studied using TGA which showed three steps; weight of loss, the first step loss up to $100^{\circ} \mathrm{C}$ is due to loss of water molecules absorbed on the surface of the St-AgNPs. St-AgNPs begin to degrade at around $250^{\circ} \mathrm{C}$. In addition, there is a steady weight loss until $550^{\circ} \mathrm{C}$.

The total weight loss up to $550^{\circ} \mathrm{C}$ for St-AgNPs (Figure 9) is about $51.17 \%$ compared to the total weight loss of St-NPs $75 \%$. The enhancement in thermal stability of St-NPs may be attributed to the significance of the surface desorption of organic compounds present in nanoparticle powder. The residue remained after cooling is pure silver bulk microstructures which possess the original color of silver. Thus, StAgNPs are expected to be made up of molecules responsible for the reduction of metal ion and stabilizing the particles in the solution.

In conclusion, St-AgNPs show decomposition profile at higher temperature than St-NPs alone, indicating improved thermal stability of St-NPs due to the presence of AgNPs.

\section{Conclusion}

For the first time, powdered starch-silver nanoparticles were synthesized having highly concentrated AgNPs with extremely small sizes by using modified nanoprecipitation method. In particular, we describe an environmental friendly one-step method to synthesize powdered AgNPs. This was done by reduction of $\mathrm{AgNO}_{3}$ solution using alkali dissolved starch meanwhile the latter acted as stabilizing agent for the formed AgNPs. Ultimately, the AgNPs in the colloidal solution that coated with starch were precipitated using the least amount of ethyl alcohol as precipitating agent. This green approach may find various medicinal applications (e.g., wound healing) as well as technological applications.

\section{Conflict of Interests}

The authors declare that there is no conflict of interests regarding the publication of this paper.

\section{References}

[1] I. O. Sosa, C. Noguez, and R. G. Barrera, "Optical properties of metal nanoparticles with arbitrary shapes," Journal of Physical Chemistry B, vol. 107, no. 26, pp. 6269-6275, 2003.

[2] D. Zhai, T. Zhang, J. Guo, X. Fang, and J. Wei, "Water-based ultraviolet curable conductive inkjet ink containing silver nanocolloids for flexible electronics," Colloids and Surfaces A, vol. 424, pp. 1-9, 2013.

[3] H. Andersson, A. Rusu, A. Manuilskiy, S. Haller, S. Ayöz, and H.-E. Nilsson, "System of nano-silver inkjet printed memory cards and PC card reader and programmer," Microelectronics Journal, vol. 42, no. 1, pp. 21-27, 2011.

[4] V. I. Pârvulescu, B. Cojocaru, V. Pârvulescu et al., "Sol-gelentrapped nano silver catalysts-correlation between active silver species and catalytic behavior," Journal of Catalysis, vol. 272, no. 1, pp. 92-100, 2010.

[5] S. Shin and J. Song, "Modeling and simulations of the removal of formaldehyde using silver nano-particles attached to granular activated carbon," Journal of Hazardous Materials, vol. 194, pp. 385-392, 2011.

[6] G. Yuan, X. Chang, and G. Zhu, "Electrosynthesis and catalytic properties of silver nano/microparticles with different morphologies," Particuology, vol. 9, no. 6, pp. 644-649, 2011.

[7] M. E. Yazdanshenas and M. Shateri-Khalilabad, "In situ synthesis of silver nanoparticles on alkali-treated cotton fabrics," Journal of Industrial Textiles, vol. 42, no. 4, pp. 459-474, 2013.

[8] D. Breitwieser, M. M. Moghaddam, S. Spirk et al., "In situ preparation of silver nanocomposites on cellulosic fibers-Microwave versus conventional heating," Carbohydrate Polymers, vol. 94, no. 1, pp. 677-686, 2013.

[9] A. Hebeish, M. E. El-Naggar, M. M. G. Fouda, M. A. Ramadan, S. S. Al-Deyab, and M. H. El-Rafie, "Highly effective antibacterial textiles containing green synthesized silver nanoparticles," Carbohydrate Polymers, vol. 86, no. 2, pp. 936-940, 2011.

[10] A.-T. Le, P. T. Huy, P. D. Tam et al., "Green synthesis of finelydispersed highly bactericidal silver nanoparticles via modified Tollens technique," Current Applied Physics, vol. 10, no. 3, pp. 910-916, 2010.

[11] M. Zheng, Z.-S. Wang, and Y.-W. Zhu, "Preparation of silver nanoparticle via active template under ultrasonic," Transactions of Nonferrous Metals Society of China, vol. 16, no. 6, pp. 13481352, 2006.

[12] G. R. Khayati and K. Janghorban, “Thermodynamic approach to synthesis of silver nanocrystalline by mechanical milling silver oxide," Transactions of Nonferrous Metals Society of China, vol. 23, no. 2, pp. 543-547, 2013.

[13] G. R. Khayati and K. Janghorban, "The nanostructure evolution of Ag powder synthesized by high energy ball milling," Advanced Powder Technology, vol. 23, no. 3, pp. 393-397, 2012.

[14] P. Mohanpuria, N. K. Rana, and S. K. Yadav, "Biosynthesis of nanoparticles: technological concepts and future applications," Journal of Nanoparticle Research, vol. 10, no. 3, pp. 507-517, 2008.

[15] U. B. Jagtap and V. A. Bapat, "Green synthesis of silver nanoparticles using Artocarpus heterophyllus Lam. seed extract and its antibacterial activity," Industrial Crops and Products, vol. 46, pp. 132-137, 2013.

[16] K. N. Thakkar, S. S. Mhatre, and R. Y. Parikh, "Biological synthesis of metallic nanoparticles," Nanomedicine, vol. 6, no. 2, pp. 257-262, 2010.

[17] S. Ghosh, S. Patil, M. Ahire et al., "Gnidia glauca flower extract mediated synthesis of gold nanoparticles and evaluation of its 
chemocatalytic potential," Journal of Nanobiotechnology, vol. 10, no. 1, p. 17, 2012.

[18] N. Vigneshwaran, R. P. Nachane, R. H. Balasubramanya, and P. V. Varadarajan, "A novel one-pot "Green" synthesis of stable silver nanoparticles using soluble starch," Carbohydrate Research, vol. 341, no. 12, pp. 2012-2018, 2006.

[19] S. Wojtysiak and A. Kudelski, "Influence of oxygen on the process of formation of silver nanoparticles during citrate/borohydride synthesis of silver sols," Colloids and Surfaces A, vol. 410, pp. 45-51, 2012.

[20] Z. Yang, H. Qian, H. Chen, and J. N. Anker, "One-pot hydrothermal synthesis of silver nanowires via citrate reduction," Journal of Colloid and Interface Science, vol. 352, no. 2, pp. 285-291, 2010.

[21] Y. Gao, P. Jiang, L. Song et al., "Studies on silver nanodecahedrons synthesized by PVP-assisted N,N-dimethylformamide (DMF) reduction," Journal of Crystal Growth, vol. 289, no. 1, pp. 376-380, 2006.

[22] R. S. Patil, M. R. Kokate, C. L. Jambhale, S. M. Pawar, S. H. Han, and S. S. Kolekar, "One-pot synthesis of PVA-capped silver nanoparticles their characterization and biomedical application," Advances in Natural Sciences, vol. 3, Article ID 015013, 7 pages, 2012.

[23] P. Raveendran, J. Fu, and S. L. Wallen, "Completely "Green" synthesis and stabilization of metal nanoparticles," Journal of the American Chemical Society, vol. 125, no. 46, pp. 13940-13941, 2003.

[24] Y.-K. Twu, Y.-W. Chen, and C.-M. Shih, "Preparation of silver nanoparticles using chitosan suspensions," Powder Technology, vol. 185, no. 3, pp. 251-257, 2008.

[25] E. S. Abdel-Halim and S. S. Al-Deyab, "Utilization of hydroxypropyl cellulose for green and efficient synthesis of silver nanoparticles," Carbohydrate Polymers, vol. 86, no. 4, pp. 1615$1622,2011$.

[26] M. H. El-Rafie, M. E. El-Naggar, M. A. Ramadan, M. M. G. Fouda, S. S. Al-Deyab, and A. Hebeish, "Environmental synthesis of silver nanoparticles using hydroxypropyl starch and their characterization," Carbohydrate Polymers, vol. 86, no. 2, pp. 630-635, 2011.

[27] P. Kouvaris, A. Delimitis, V. Zaspalis, D. Papadopoulos, S. A. Tsipas, and N. Michailidis, "Green synthesis and characterization of silver nanoparticles produced using Arbutus Unedo leaf extract," Materials Letters, vol. 76, pp. 18-20, 2012.

[28] J. Eastman, "Stability of charge stabilized colloids," in Colloid Science: Principles, Methods and Applications, T. Cosgrove, Ed., p. 54, Blackwell UK, 2005.

[29] O. Choi and Z. Hu, "Size dependent and reactive oxygen species related nanosilver toxicity to nitrifying bacteria," Environmental Science and Technology, vol. 42, no. 12, pp. 4583-4588, 2008.

[30] S. Pandey, G. K. Goswami, and K. K. Nanda, "Green synthesis of biopolymer-silver nanoparticle nanocomposite: an optical sensor for ammonia detection," International Journal of Biological Macromolecules, vol. 51, no. 4, pp. 583-589, 2012. 

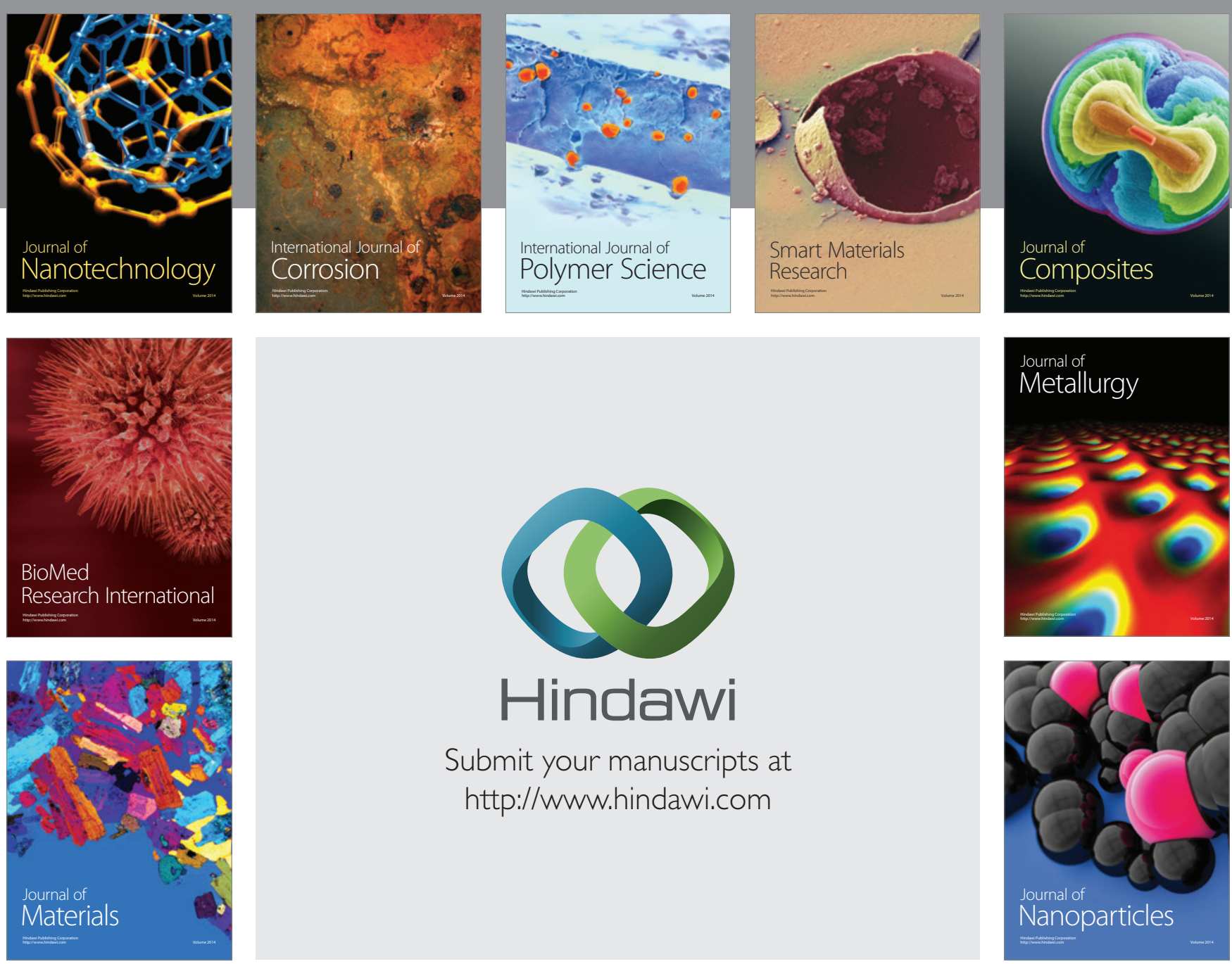

Submit your manuscripts at http://www.hindawi.com
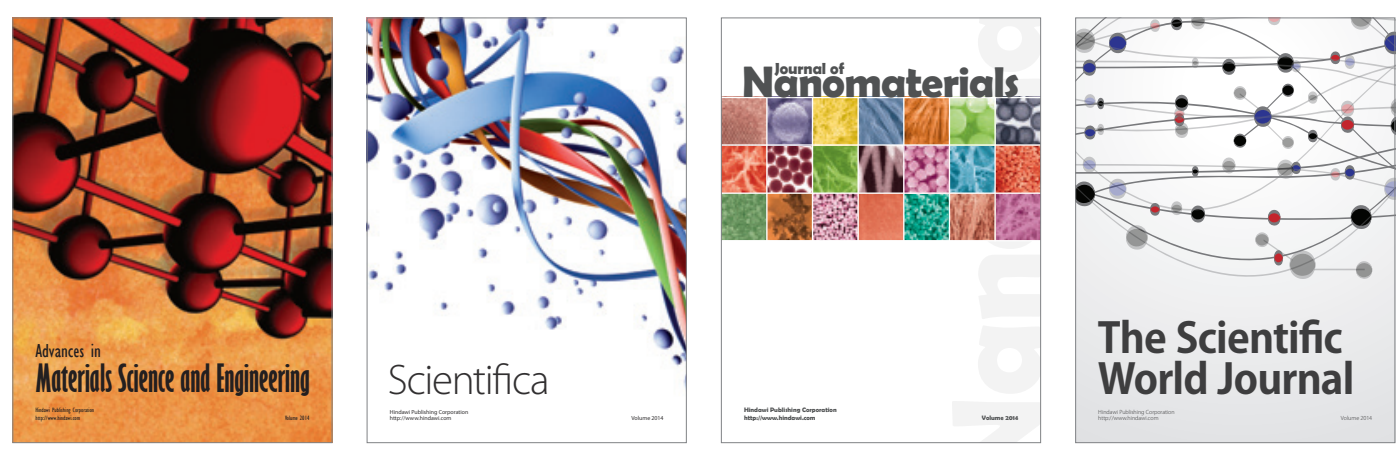

\section{The Scientific World Journal}
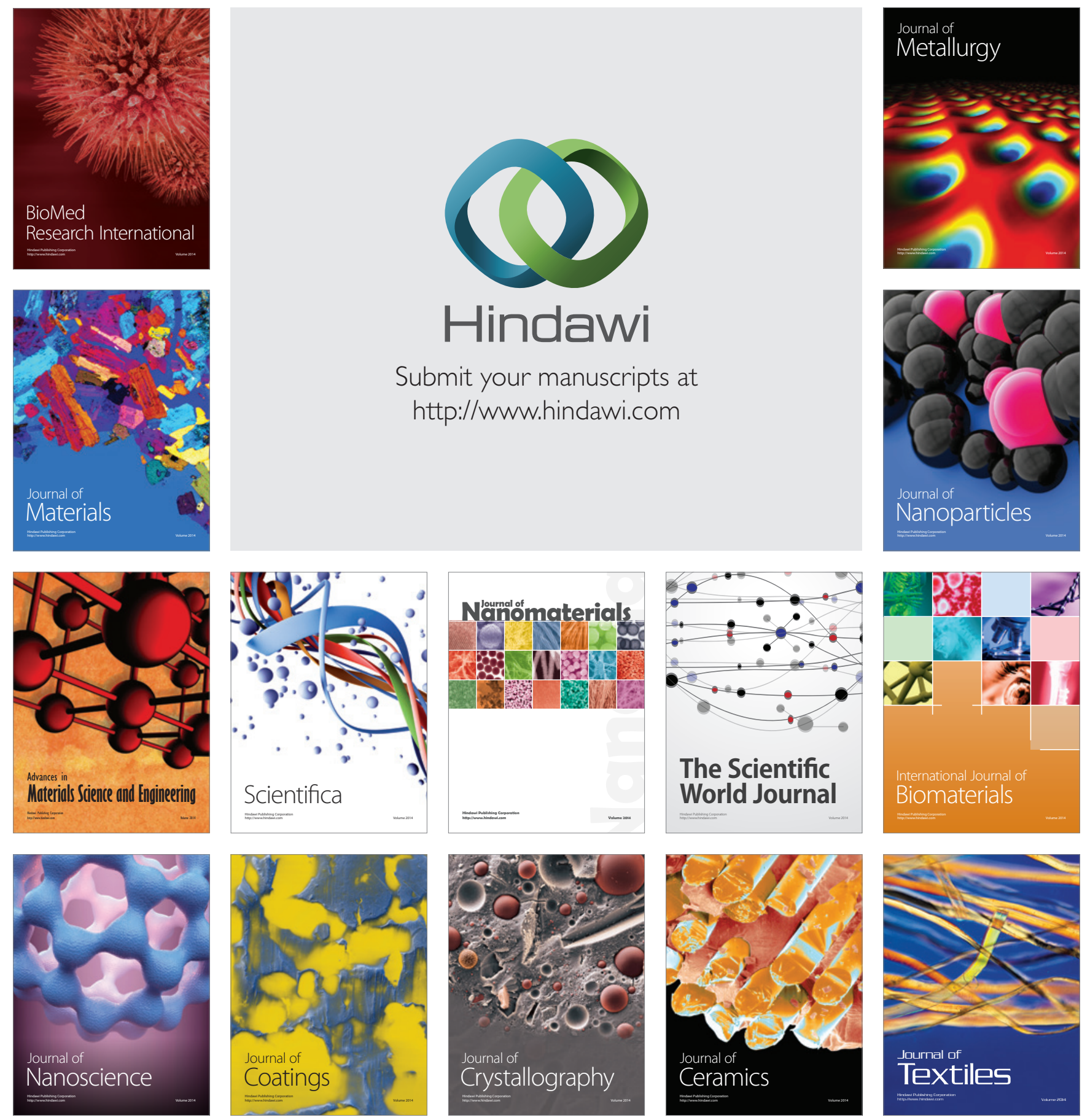